Check for updates

Cite this: RSC Adv., 2019, 9, 6986

Received 3rd January 2019

Accepted 12th February 2019

DOI: $10.1039 / c 9 r a 00048 h$

rsc.li/rsc-advances

\section{A green L-cysteine modified cellulose nanocrystals biosorbent for adsorption of mercury ions from aqueous solutions $\uparrow$}

\begin{abstract}
Weixue Li, Benzhi Ju (D) * and Shufen Zhang (D)
Using a green biosorbent to remove toxic mercury ions from aqueous solutions is a significant undertaking. In the present study, a novel biosorbent, L-cysteine modified cellulose nanocrystals (Lcys-CNCs), was prepared by functionalizing high surface area cellulose nanocrystals with L-cysteine through periodate oxidation and reductive amination reaction. Lcys-CNCs were characterized by FT-IR, ${ }^{13} \mathrm{C} C P-M A S ~ N M R$, elemental analysis, XPS, zeta potential and SEM. As cellulose nanocrystals are the natural nanomaterial, and L-cysteine contains strong mercury chelating groups, Lcys-CNCs show excellent adsorption capacity for mercury ions. The experimental conditions such as $\mathrm{pH}$, contact time, and initial mercury ion concentration are discussed. The pseudo-second order model can describe the removal kinetics of $\mathrm{Hg}(॥)$ more accurately than the pseudo-first order model. The adsorption isotherm study of $\mathrm{Hg}(॥)$ followed the Langmuir model of monolayer adsorption. The maximum uptake capacity of Lcys-CNCs was determined to be $923 \mathrm{mg} \mathrm{g}^{-1}$. Lcys-CNCs can remove mercury ions with $93 \%$ removal efficiency within $5 \mathrm{~min}$ from a $71 \mathrm{mg} \mathrm{L}^{-1}$ solution. For $\mathrm{Cd}(॥), \mathrm{Pb}(॥), \mathrm{Cu}(॥)$ and $\mathrm{Zn}(॥)$ ions, Lcsy-CNCs can selectively adsorb $\mathrm{Hg}(॥)$ ions and the removal efficiency is $87.4 \%$ for $\mathrm{Hg}(॥)$. This study suggests Lcsy-CNCs are a green and highly efficient biosorbent for adsorption of mercury ions from aqueous solutions.
\end{abstract}

\section{Introduction}

Mercury is a common heavy metal that is toxic to living organisms. In recent decades, the development of modern industry has caused mercury pollution to a great extent. ${ }^{1,2}$ The accumulation of mercury in human bodies can cause serious health issues. ${ }^{3}$ Therefore, the removal of $\mathrm{Hg}$ (II) pollutants from water is required to reduce the harm of $\mathrm{Hg}$ (II) to organisms. ${ }^{4}$ Among various treatment methods, adsorption is an effective, simple and feasible method to remove $\mathrm{Hg}$ (II) from water. ${ }^{5}$ At present, many adsorbents (such as inorganic $^{6-8}$ and organic materials ${ }^{9}$ ) have been developed to remove $\mathrm{Hg}$ (II) from aqueous solutions. However, their application is limited because they are not renewable or cause secondary pollution to the environment. According to the concept of sustainable development, it is important to develop a green biosorbent ${ }^{\mathbf{1 0}}$ based on biomass that can efficiently and selectively remove mercury ions. Biosorbents are more favored than traditional adsorbents, due to their less toxicity, wide sources and biodegradation.11,12 Commonly used biosorbents include cellulose-based adsorbents, ${ }^{13-15}$ chitosan-based adsorbents, ${ }^{16,17}$ and Agaricus macrospores ${ }^{18}$ etc. However, these reported biosorbents still face many

State Key Laboratory of Fine Chemicals, Dalian University of Technology, Dalian 116024, PR China. E-mail: jubenzhi@dlut.edu.cn; Fax: +86 411 84986264; Tel: +86 41184986269

† Electronic supplementary information (ESI) available. See DOI: 10.1039/c9ra00048h challenges such as low specific surface area, less mercury chelating groups and poor selective adsorption of mercury ions.

Cellulose is the most abundant biopolymer in nature. And its nanoform, cellulose nanocrystals (CNCs), can be used as a promising adsorbent due to their sustainability, biodegradability, biocompatibility and high specific surface properties. ${ }^{\mathbf{1 9}}$ However, the adsorption capacity of CNCs is not high due to the lack of strong mercury chelating groups.

The introduction of functional groups (e.g. amino group, thiol group) that have strong affinity to $\mathrm{Hg}$ (II) ions on the surface of adsorbents can improve their adsorption capacity for mercury ions. ${ }^{20}$ Liu and co-workers ${ }^{21}$ reported that maximum adsorption capacity was $718 \mathrm{mg} \mathrm{g}^{-1}$ by 3-mercaptopropyltrimethoxysilane (MPTs) modified TO-NFC aerogel. Zhang and co-workers $^{22}$ reported that the maximal uptake capacity as high as $543 \mathrm{mg} \mathrm{g}^{-1}$ by triethylenetetramine (TETA) functionalized magnetic poly(glycidyl methacrylate) (PGMA) nano-adsorbent. Therefore, increasing the content of amino and thiol group on the CNC can help increase its adsorbability of mercury ions.

L-Cysteine is a common amino acid in the body, and contains strong mercury chelating groups such as thiol group, amino group. The L-cystine functionalized-exfoliated graphene oxide adsorbent have been used for removal of $\mathrm{Hg}$ (II). ${ }^{23}$ L-Cysteine doped polypyrrole (PPy@L-Cyst) have been reported to be rapid and efficient removal of $\mathrm{Hg}$ (II) ions from water. ${ }^{24}$ Thus, the content of the thiol group and the amino group on the surface of the CNCs is increased by grafting ${ }_{\mathrm{L}}$-cysteine. 
In this study, a green biosorbent, L-cysteine modified cellulose nanocrystals, was successfully synthesized by periodate oxidation and reductive amination reaction. Lcys-CNCs have the following features: renewability because the raw material is green biomass; high specific surface; a large number of mercury chelating groups (thiol and amino groups) that are anchored to CNCs by covalent bond. The effect of $\mathrm{pH}$, contact time, mercury ions initial concentration was thoroughly investigated to optimize the adsorption process. Herein, Lcys-CNCs exhibited the excellent adsorption capacity for mercury ions and could quickly achieve adsorption equilibrium. Lcys-CNCs can selectively adsorb mercury ions in the presence of other coexisting metal ions, and show a good regeneration performance.

\section{Experimental}

\subsection{Chemicals and reagents}

Qualitative filter paper (Hangzhou Fuyang Special Paper Industry Co., Ltd.) were used as CNC source. Sulfuric acid $\left(\mathrm{H}_{2} \mathrm{SO}_{4}, 98 \%\right)$, sodium periodate $\left(\mathrm{NaIO}_{4}\right)$, glycol, L-cysteine and sodium cyanoborohydride $\left(\mathrm{NaBH}_{3} \mathrm{CN}\right)$ were purchased from Sigma-Aldrich.

\subsection{Synthesis of dialdehyde cellulose nanocrystal}

CNCs were synthesis by $\mathrm{H}_{2} \mathrm{SO}_{4}$ hydrolysis of filter paper. The powdered filter paper was added slowly into sulfuric acid (64 wt\%, $10 \mathrm{~mL} \mathrm{~g}^{-1}$ ) under mechanical stirring at $45{ }^{\circ} \mathrm{C}$ for $45 \mathrm{~min}$, then deionized water $\left(4^{\circ} \mathrm{C}, 10\right.$ equivalents per $\mathrm{mL}$ of the reaction solution) was added into reaction. CNCs were separated from the resulting suspension by centrifugation (9000 rpm, $5 \mathrm{~min}$ ). The sediment was dialyzed with the dialysis membranes (molecular weight cut-off, MWCO: 8000-14 000) against deionized water to neutrality. Then the suspension could remove aggregates by centrifugation ( $8000 \mathrm{rpm}, 10 \mathrm{~min}$ ). The CNC suspension (2.0 wt\%) and sodium periodate (corresponding to $6.0 \mathrm{mmol} \mathrm{g}^{-1} \mathrm{CNCs}$ ) were mixed for $12 \mathrm{~h}$ in the absence of light at $40{ }^{\circ} \mathrm{C}$. At the end the oxidation period, $10 \mathrm{~mL}$ ethylene glycol was added into the reaction mixture to remove the residual sodium periodate. The resulting product was dialyzed (MWCO: 8000-14 000) against deionized water to that of deionized water $\left(\sim 5 \mu \mathrm{S} \mathrm{cm}^{-1}\right)$. The resulting dialdehyde cellulose (DAC) nanocrystal was stored at $5{ }^{\circ} \mathrm{C} .{ }^{25,26}$

The aldehyde content of DAC was measured by the Schiff base reaction between aldehyde groups and hydroxylamine hydrochloride. ${ }^{27,28}$ In this work, aldehyde content of DAC was $3.49 \mathrm{mmol} \mathrm{g}^{-1}$.

\subsection{Synthesis of Lcys-CNCs}

L-Cysteine $(9.51 \mathrm{~g})$ was slowly added to DAC $(300 \mathrm{~mL}, 0.015 \mathrm{~g}$ $\mathrm{mL}^{-1}$ ) suspension, then the reaction was stirred for $6 \mathrm{~h}$ at room temperature. $\mathrm{NaBH}_{3} \mathrm{CN}$ (1.97 g) was added in multiple steps into reaction and continued for $6 \mathrm{~h}$ at room temperature, followed by addition of $\mathrm{HCl}$ dilute solution $\left(3 \mathrm{~mol} \mathrm{~L}^{-1}\right)$ to neutralize the excess $\mathrm{NaBH}_{3} \mathrm{CN}$. The resulting product was dialyzed (MWCO: 8000-14 000) against deionized water to that of deionized water $\left(\sim 5 \mu \mathrm{S} \mathrm{cm}^{-1}\right)$, then freeze dried.

\subsection{Characterization}

Characterization is shown in ESI. $\dagger$

\subsection{Adsorption/desorption studies}

Batch adsorption experiments were conducted by a certain amount of dry Lcys-CNCs $(\sim 10 \mathrm{mg})$ were added into $30 \mathrm{~mL}$ $\mathrm{Hg}$ (II) solution, at different $\mathrm{Hg}$ (II) concentration (71-502 $\mathrm{mg} \mathrm{L}^{-1}$ ) and contact time $(0.5-180 \mathrm{~min})$, the varying $\mathrm{pH}$ value $(2-7)$, and constant $25{ }^{\circ} \mathrm{C}^{29}$ Afterwards, the precipitate was separated by centrifugation (5000 rpm, $10 \mathrm{~min}$ ) and the amount of mercury in the solution was quantified by ICP-MS. In addition, the selective removal of $\mathrm{Hg}$ (II) from a solution containing $\mathrm{Hg}$ (II) at $116 \mathrm{mg} \mathrm{L}^{-1}$ and other toxic metals $\mathrm{Pb}$ (II), $\mathrm{Cd}$ (II), $\mathrm{Zn}$ (II) and $\mathrm{Cu}$ (II) at 90, 80, 108 and $91 \mathrm{mg} \mathrm{L}^{-1}$, respectively, was evaluated at pH 5 and $25{ }^{\circ} \mathrm{C}$ for $3 \mathrm{~h}$. Desorption was carried out using Lcys-CNCs preloaded with $\mathrm{Hg}$ (II) in mercury ion solution. Briefly, a known amount of the loaded Lcys-CNCs were eluted with $2 \mathrm{M} \mathrm{HCl}$ and $0.5 \mathrm{M}$ thiourea by stirring for $30 \mathrm{~min}$ at $25{ }^{\circ} \mathrm{C}$. For the regeneration study of Lcys-CNCs, this adsorption-desorption cycle was repeated four times. The amount of mercury adsorbed per gram and removal efficiency of Lcys-CNCs was calculated according to following equations. ${ }^{30,31}$

$$
\begin{gathered}
Q_{\mathrm{e}}=\frac{\left(C_{\mathrm{o}}-C_{\mathrm{e}}\right) V}{m} \\
\text { Removal efficiency }=\frac{C_{\mathrm{o}}-C_{\mathrm{e}}}{C_{\mathrm{o}}} \times 100 \%
\end{gathered}
$$

where $Q_{\mathrm{e}}$ is the adsorption capacity of Lcys-CNCs $\left(\mathrm{mg} \mathrm{g}^{-1}\right)$ at equilibrium, $C_{\mathrm{o}}$ is the initial concentration of mercury $\left(\mathrm{mg} \mathrm{L}^{-1}\right)$, $C_{\mathrm{e}}$ is in the concentration of the mercury ion $\left(\mathrm{mg} \mathrm{L}^{-1}\right)$ at equilibrium, $V$ is the volume of the solution (L), and $m$ is the weight of Lcys-CNCs (g).

\section{Results and discussion}

\subsection{Synthesis and characterization}

CNCs were prepared by $\mathrm{H}_{2} \mathrm{SO}_{4}$ (64 wt\%) hydrolysis from filter paper after which rod-shaped crystalline region (CNC) remained while the amorphous region was removed. Sodium periodate could selectively oxidize $\mathrm{CNC}$ and cleave the $\mathrm{C} 2-\mathrm{C} 3$ bond to synthesize DAC. DAC was then grafted with $\mathrm{L}$-cysteine to obtain Lcys-CNCs through reductive amination reaction (Scheme 1).

The molecular structure of Lcsy-CNCs was determined by FTIR, XPS and solid-state ${ }^{13} \mathrm{C}$ NMR. The FT-IR spectra of CNCs, DAC and Lcsy-CNCs was shown in Fig. 1A. As shown in Fig. 1A, CNCs, DAC and Lcys-CNCs had the same absorption bands at $3418,1634,1436,1318,1164$ and $665 \mathrm{~cm}^{-1}$ correspond to the $\mathrm{OH}$ stretching vibration, $\mathrm{OH}$ bending vibration of absorbed water, the $\mathrm{HCH}$ and $\mathrm{OCH}$ in-plane bending vibration, $\mathrm{CH}_{2}$ rocking vibration at the $\mathrm{C}_{6}$ position, $\mathrm{C}-\mathrm{O}-\mathrm{C}$ asymmetrical stretching and $\mathrm{C}-\mathrm{OH}$ out-of-plane bending, respectively. ${ }^{32}$ These absorption bands indicate that the CNCs, DAC and LcysCNCs have characteristic bands of cellulose. Compared to CNCs, the characteristic absorption band appeared at $1730 \mathrm{~cm}^{-1}$ for the stretching vibration of the $\mathrm{C}=\mathrm{O}$ of $\mathrm{DAC}$, 


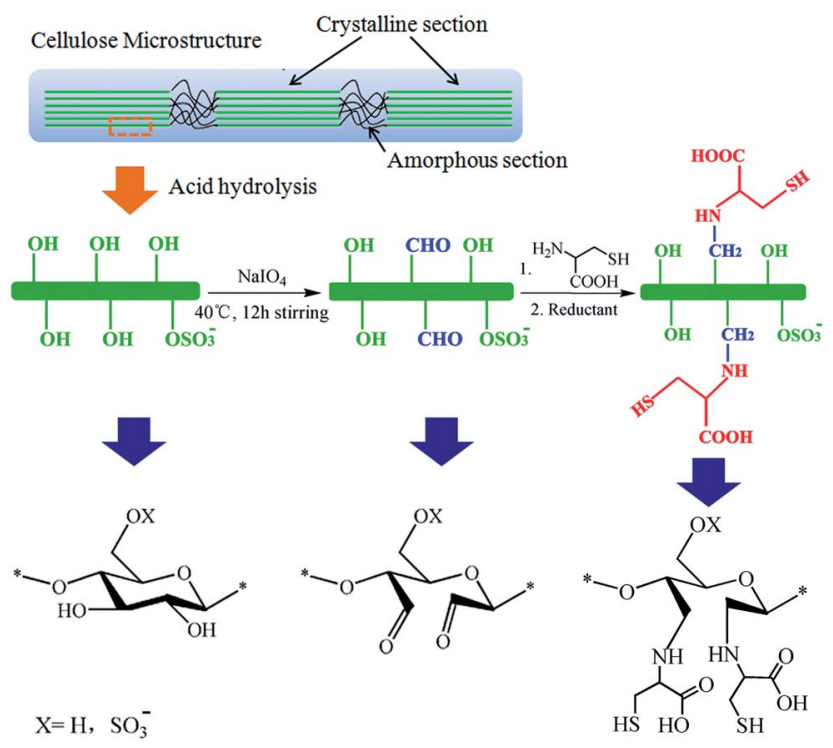

Scheme 1 Oxidation and reduction reaction of cellulose nanocrystals.

which is caused by the formation of aldehyde groups. The two absorption bands at 1731 and $2550 \mathrm{~cm}^{-1}$ in the spectrum of Lcys-CNCs correspond to $\mathrm{COOH}$ and $\mathrm{S}-\mathrm{H}$ groups, respectively. This result indicated that I-cysteine was grafted onto CNCs surface.

XPS was used to further study the surface chemical composition of Lcsy-CNCs. As shown in Fig. 1B, compared to CNCs, Lcys-CNCs shown new peaks appeared in the $\mathrm{N} 1 \mathrm{~s}$ and $\mathrm{S} 2 \mathrm{p}$ regions. The S 2p XPS spectrum of Lcsy-CNCs (Fig. 1C, Table S1†) displayed two characteristic peaks, one of which for sulfate (high oxidation state sulfur, S 2p 3/2 at $167.0 \mathrm{eV}$ and S 2p 1/2 at $168.4 \mathrm{eV}$ ) and the other for thiol groups (S 2p 3/2 at $161.6 \mathrm{eV}$ and $\mathrm{S} 2 \mathrm{p} 1 / 2$ at $162.8 \mathrm{eV}) .{ }^{33}$ At the same time, the $\mathrm{N} 1 \mathrm{~s}$ XPS spectrum of Lcsy-CNCs (Fig. 1D) shown two characteristic peaks, one of which $(397.8 \mathrm{eV})$ was assigned to amino groups and one of which $(400.1 \mathrm{eV})$ was assigned to the protonatable amino groups (amino groups were protonated when $\mathrm{HCl}$ was added to remove unreacted $\mathrm{NaBH}_{3} \mathrm{CN}$ ). The C 1s XPS spectrum of LcsyCNCs (Fig. 1E) shown four peaks, which were attributed to $\mathrm{C}-\mathrm{C}(283.8 \mathrm{eV}), \mathrm{C}-\mathrm{O}(284.7 \mathrm{eV}), \mathrm{O}-\mathrm{C}-\mathrm{O}(286.0 \mathrm{eV})$ and $\mathrm{O}-\mathrm{C}=\mathrm{O}$ $(286.8 \mathrm{eV})$, respectively. Moreover, the O 1s XPS spectrum of Lcsy-CNC (Fig. 1F) displayed two splitting peaks of the binding energy at 530.0 and $531.1 \mathrm{eV}$ could be assigned to $\mathrm{C}-\mathrm{O}-\mathrm{H}$ and $\mathrm{O}-\mathrm{C}-\mathrm{O}$ or $\mathrm{C}=\mathrm{O}^{34}$

The structure of Lcys-CNCs was also analyzed with the solidstate ${ }^{13} \mathrm{C}$ NMR. As can be seen in Fig. $1 \mathrm{G}-\mathrm{H}$, the ${ }^{13} \mathrm{C}$ CP-MAS NMR spectra of CNCs and Lcys-CNCs displayed typical signals from cellulose, C1 (105 ppm), C2, C3 and C5 (70-80 ppm), C4 ( $\sim 89 \mathrm{ppm})$, and C6 ( 65 ppm) ${ }^{35,36}$ Moreover, ${ }^{13} \mathrm{C}$ CP-MAS NMR spectra of Lcys-CNCs (Fig. 1H) showed peaks at $32 \mathrm{ppm}$ (C8 carbons, $-\mathrm{C}-\mathrm{S}) 54 \mathrm{ppm}(\mathrm{C} 7$ carbons, $-\mathrm{C}-\mathrm{N})$ and $174 \mathrm{ppm}$ (C9 carbons, $-\mathrm{C}=\mathrm{O}){ }^{37,38}$

The contents of surface $\mathrm{S}$ and $\mathrm{N}$ of the adsorbent directly affect its adsorption capacity of $\mathrm{Hg}$ (II). ${ }^{21}$ The proportions of $\mathrm{S}$ and $\mathrm{N}$ in CNCs and Lcys-CNCs are performed by elemental analysis in Table 1 . The calculated content of surface $\mathrm{S}$ and $\mathrm{N}$ elements in Lcys-CNCs was 2.80 and $2.77 \mathrm{mmol} \mathrm{g}^{-1}$, respectively. Therefore, the nitrogen and sulfur contents of Lcys-CNCs were significantly increased after CNCs modification by $\mathrm{L}^{-}$ cysteine. The specific surface area of adsorbent is also a major factor affecting the adsorption performance. The $\mathrm{N}_{2}$ adsorption-desorption isotherms of CNCs and Lcys-CNCs are presented in Fig. S1. $\dagger$ The BET specific surface area of CNCs and Lcys-CNCs shown in the Table 1, reveal that Lcys-CNCs $(72.10$ $\mathrm{m}^{2} \mathrm{~g}^{-1}$ ) have a much higher BET surface area than that of CNCs $\left(10.81 \mathrm{~m}^{2} \mathrm{~g}^{-1}\right)$ due to the destruction of the ordered packing of CNCs by sodium periodate oxidation and post-modification reactions, which loosens its surface.

Thus, the factors affecting the adsorption capacity of mercury ions include specific surface area and mercury chelating groups. Lcys-CNCs are the nano-material with higher specific surface area. A large number of amino and thiol groups were introduced on the surface of CNCs by chemical modification. Therefore, Lcys-CNCs with the above structural characteristics implies it can efficiently adsorb mercury ions.

\subsection{Adsorption studies}

To evaluate the adsorption capacity of Lcsy-CNCs for removing $\mathrm{Hg}$ (II), a certain amount of dry Lcys-CNCs were added into $30 \mathrm{~mL} \mathrm{Hg}$ (II) solution at $25^{\circ} \mathrm{C}$. The $\mathrm{pH}$ value of solution can change the surface charge density of adsorbent, and influences the adsorption property of $\mathrm{Hg}$ (II). Therefore, the effect of $\mathrm{pH}$ value on the adsorption capacity of $\mathrm{Hg}$ (II) by Lcys-CNCs (Fig. 2a) and the effect of zeta potential of Lcys-CNCs at different $\mathrm{pH}$ values (Fig. 2b) were studied.

When CNCs were used as adsorbent, the adsorption was very low, and the $\mathrm{pH}$ value had a mild influence on the adsorption. However, after the L-cysteine was introduced on CNCs, the adsorption capacity of $\mathrm{Hg}$ (II) was greatly increased, and the adsorption capacity also changed at different $\mathrm{pH}$ values. When the $\mathrm{pH}$ was raised from 2 to 5 , the adsorption of mercury ions by Lcys-CNCs showed an upward trend. At $\mathrm{pH}$ 5, it reached the maximum, but the adsorption properties decreased with $\mathrm{pH}$ value $(>5)$ increased.

The reason why the uptake capacity of Lcys-CNCs was affected by $\mathrm{pH}$ is as follows. At $\mathrm{pH} 1.56$, the zeta potential of Lcys-CNCs was $+4.39 \mathrm{mV}$ due to the -NH- groups of Lcys-CNCs were protonated. At the same time, the protons compete with $\mathrm{Hg}$ (II) ions for getting adsorbed onto active sites of adsorbent and electrostatic repulsion between mercury ions and LcysCNCs results in decreasing adsorption of $\mathrm{Hg}$ (II). ${ }^{39}$ The zeta potential of Lcys-CNCs decreases rapidly with increasing $\mathrm{pH}$ value, because the deprotonation of the amine and carboxyl groups on the surface of Lcys-CNC at high $\mathrm{pH}$. At $\mathrm{pH} 5, \mathrm{~L}^{-}$ cysteine reaches the isoelectric point, the number of protonated amino groups and deprotonated carboxyl groups is equal, and $\mathrm{L}^{-}$ cysteine is uncharged. ${ }^{40}$ However, Lcys-CNC is obtained by introducing L-cysteine into the surface of the CNC, and its surface contains not only amino, carboxyl groups but also sulfate groups. Therefore, the potential of Lcys-CNC is $-28.3 \mathrm{mV}$ at $\mathrm{pH}$ 5.31, and Lcys-CNC has strong electrostatic attraction to $\mathrm{Hg}(\mathrm{II})$ and can quickly, effectively adsorb $\mathrm{Hg}$ (II) 
(A)

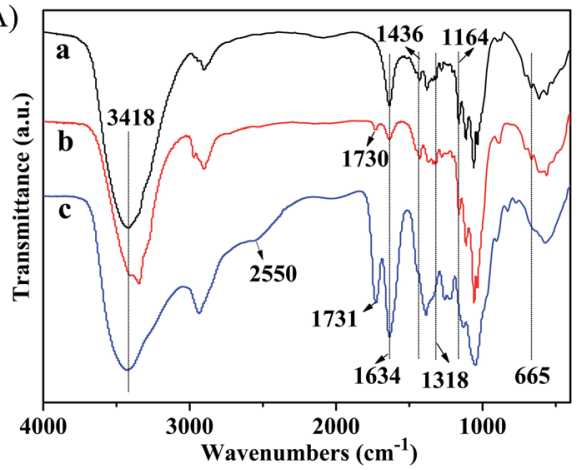

(B)
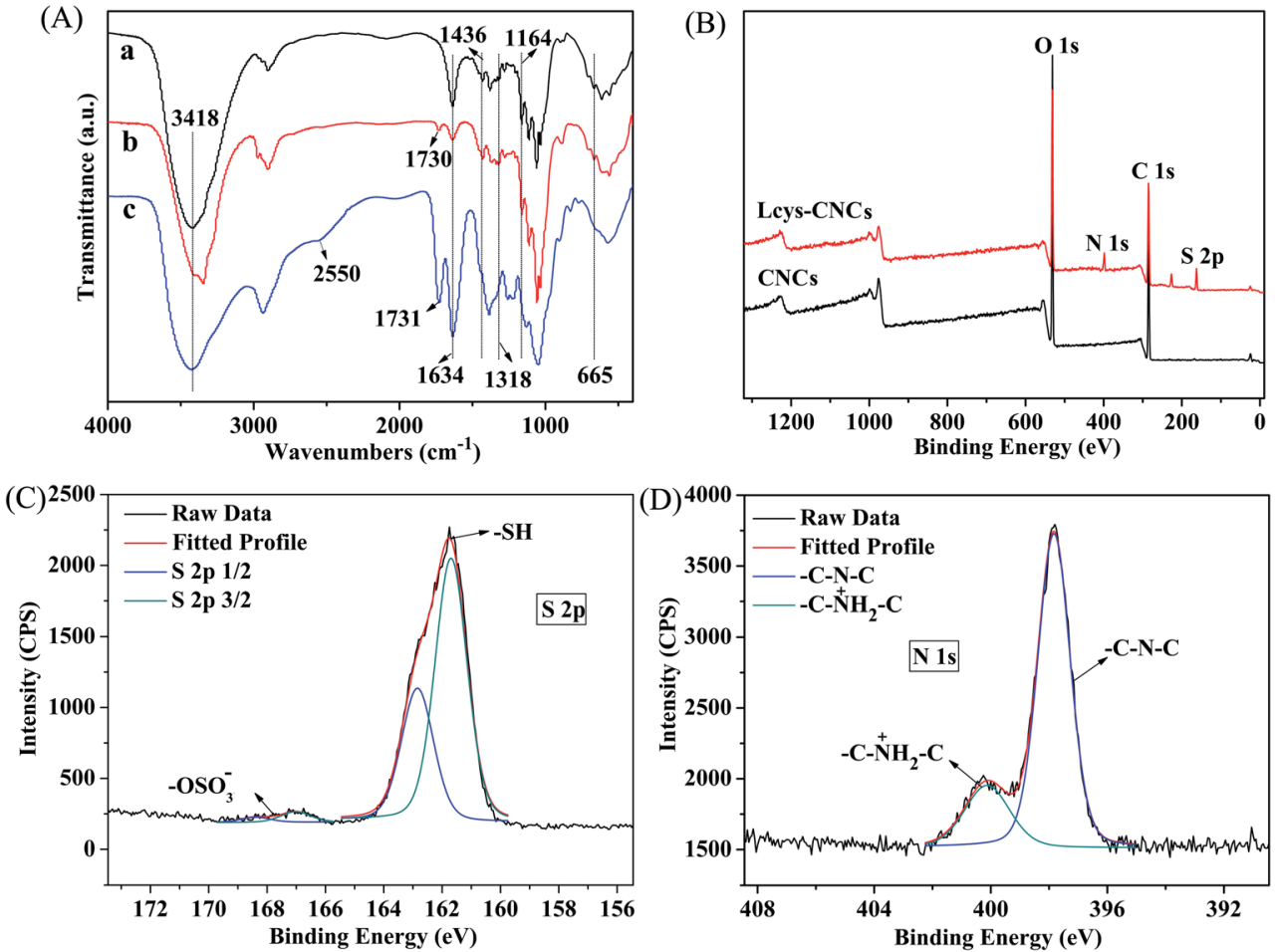

(E)

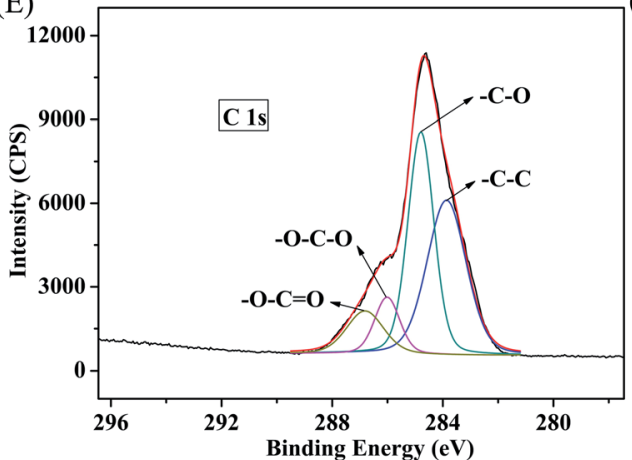

(G)

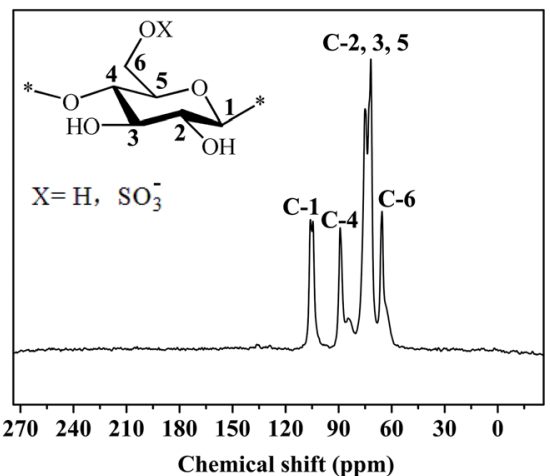

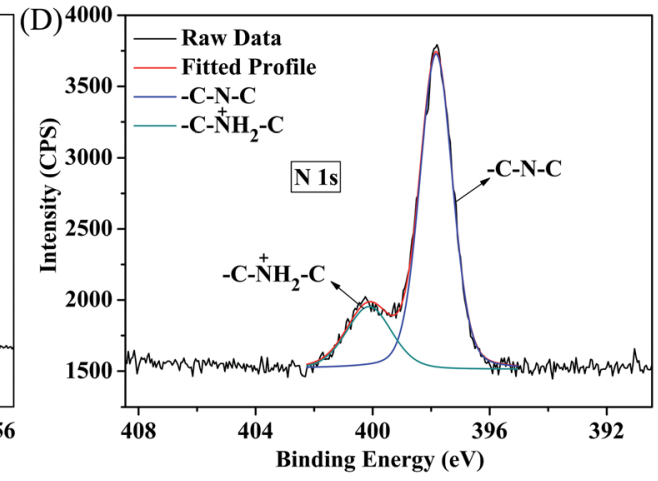

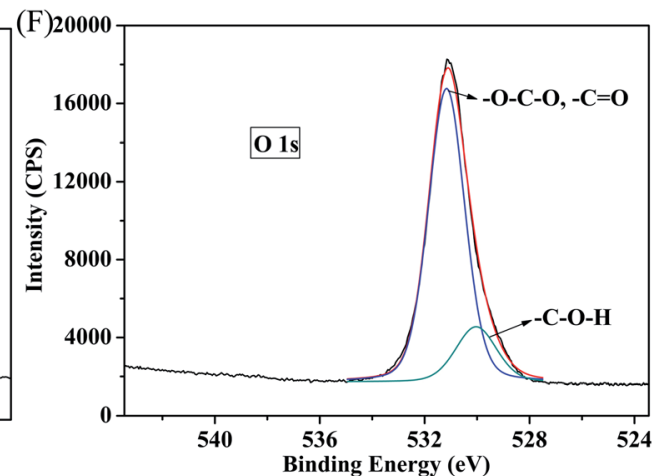

(H)

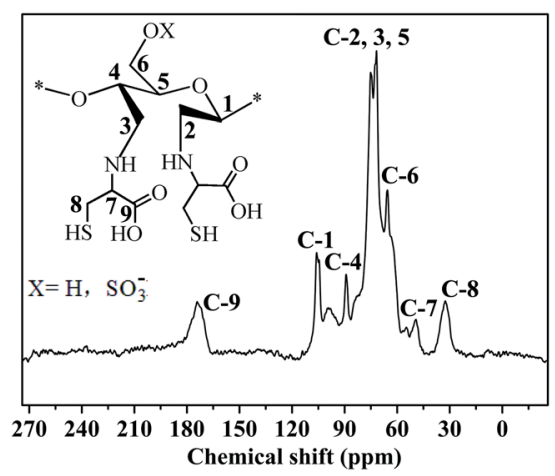

Fig. 1 (A) The FT-IR spectra of CNCs (a), DAC (b) and Lcys-CNCs (c); (B) XPS survey spectra of CNCs and Lcys-CNCs. The S 2p XPS spectrum (C), N 1s XPS spectrum (D), C 1s XPS spectrum (E) and O 1s XPS spectrum (F) of Lcys-CNC; ${ }^{13} \mathrm{C} \mathrm{CP-MAS} \mathrm{NMR} \mathrm{spectra} \mathrm{of} \mathrm{CNCs} \mathrm{(G)} \mathrm{and} \mathrm{Lcys-CNCs} \mathrm{(H).}$

from aqueous solutions. Lcys-CNCs reach the maximum uptake capacity $\left(587 \mathrm{mg} \mathrm{g}^{-1}\right)$ at $\mathrm{pH} 5$ (Fig. 2a). Although the zeta potential of Lcys-CNCs decreases with $\mathrm{pH}$ value $(>5)$ increases, the amount of $\mathrm{OH}^{-}$increases, and $\mathrm{OH}^{-}$groups react with $\mathrm{Hg}$ (II) ions to form $\mathrm{Hg}(\mathrm{OH})^{+}, \mathrm{Hg}(\mathrm{OH})_{2}$, and $\mathrm{Hg}(\mathrm{OH})_{3}{ }^{-}$, which reduces the adsorption effect. ${ }^{41}$

\subsection{Adsorption kinetics}

When the adsorbent reached the adsorption equilibrium, the required contact time can evaluate the adsorbent. After LcysCNCs were added to the mercury ion solution, the adsorption capacity of mercury ion changed with the contact time. ${ }^{42}$ The influence of contact time on the uptake of mercury ions onto 
Table 1 Composition of CNCs and Lcys-CNCs measured by elemental analysis; content of surface S element, surface N element and BET surface area of the samples

\begin{tabular}{|c|c|c|c|c|c|c|c|}
\hline Samples & $\% \mathrm{C}$ & $\% \mathrm{H}$ & $\% \mathrm{~S}$ & $\% \mathrm{~N}$ & $\begin{array}{l}\text { Surface } \\
\frac{\mathrm{S}}{\mathrm{mmol} \mathrm{g}^{-1}}\end{array}$ & $\begin{array}{l}\text { Surface } \\
\frac{\mathrm{N}}{\mathrm{mmol} \mathrm{g}^{-1}}\end{array}$ & $\begin{array}{l}\begin{array}{l}\text { BET surface } \\
\text { area }\end{array} \\
\mathrm{m}^{2} \mathrm{~g}^{-1}\end{array}$ \\
\hline CNCs & 41.36 & 6.70 & 0.89 & 0.29 & 0.27 & 0.20 & 10.81 \\
\hline Lcsy-CNCs & 41.85 & 6.15 & 8.98 & 3.89 & 2.80 & 2.77 & 72.10 \\
\hline
\end{tabular}
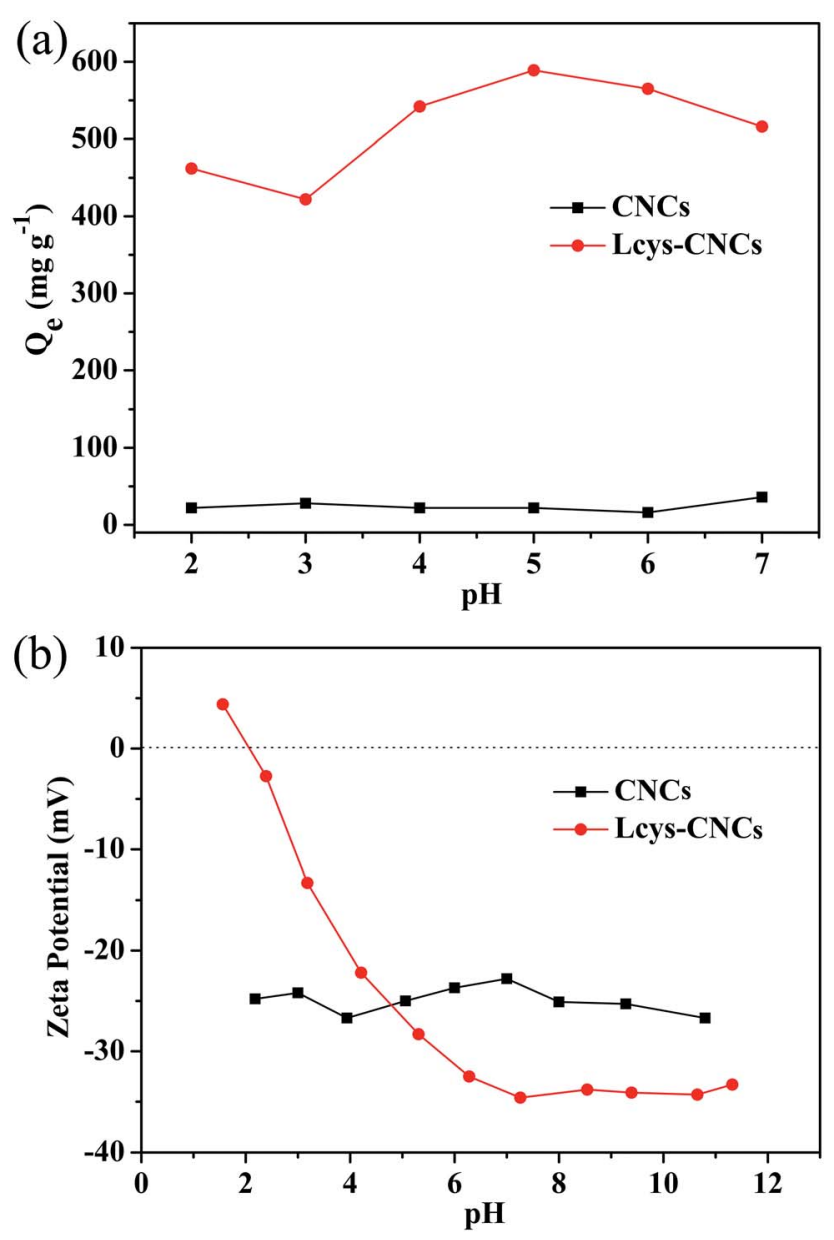

Fig. 2 (a) Effect of $\mathrm{pH}$ value on the adsorption capacity of $\mathrm{Hg}(॥)$ (215 mg L ${ }^{-1}, 25^{\circ} \mathrm{C}, 3 \mathrm{~h}, \mathrm{pH} 2-7$ ). (b) Zeta potential of CNCs and Lcys$\mathrm{CNCs}$ at different $\mathrm{pH}$ values $\left(1.0 \mathrm{~g} \mathrm{~L}^{-1}, 25^{\circ} \mathrm{C}, 0.01 \mathrm{M} \mathrm{KCl}\right)$.

Lcys-CNCs (Fig. 3a) was investigated in $30 \mathrm{~mL}$ of $71 \mathrm{mg} \mathrm{L^{-1 }}$ $\mathrm{Hg}$ (II) solution ( $\mathrm{pH} 5$ ) at $25{ }^{\circ} \mathrm{C}$. When Lcys-CNCs were added to the mercury ion solution, mercury ions could be quickly and efficiently adsorbed from aqueous solution because of LcysCNCs with high specific surface area and a large number of active sites. In particular, the negative charge of Lcys-CNCs could further accelerate the arrival of $\mathrm{Hg}$ (II) ions on the surface of the adsorbent by electrostatic interaction. ${ }^{21}$ Afterword, the active sites decreased due to mercury ions accumulated on the surface of Lcys-CNCs. Therefore, the adsorption rate gradually slowed down, then achieved adsorption equilibrium within $5 \mathrm{~min}$.
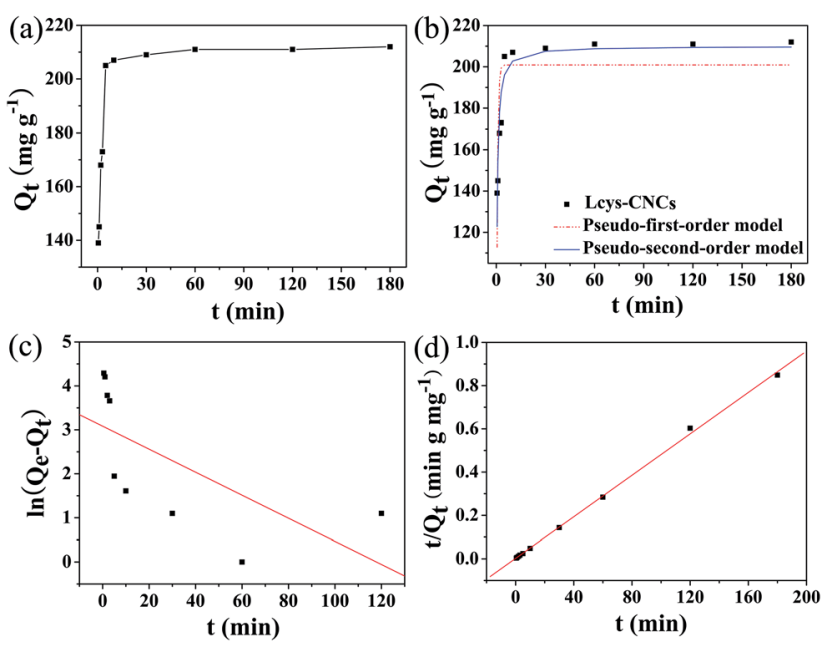

Fig. 3 Effect of contact time on the adsorption capacity of $\mathrm{Hg}($ (I) (a); nonlinear forms of pseudo-first-order model and pseudo-secondorder model for adsorption of Hg(॥) by Lcys-CNCs (b); linear forms of pseudo-first-order model (c) and pseudo-second-order model (d) (71 $\mathrm{mg} \mathrm{L}^{-1}, \mathrm{pH} 5,25^{\circ} \mathrm{C}, 0.5-180 \mathrm{~min}$ ).

The adsorption kinetic study can explain the adsorption mechanism. Therefore, pseudo-first-order and pseudo-secondorder kinetic models (Fig. 3b-d) were applied to describe the adsorption of mercury ions onto Lcys-CNCs, and their models were shown as the following equations.

Nonlinear forms of pseudo-first-order:

$$
Q_{t}=Q_{\mathrm{e}}\left[1-\exp \left(-k_{1} t\right)\right]
$$

Linear forms of pseudo-first-order:

$$
\ln \left(Q_{\mathrm{e}}-Q_{t}\right)=\ln Q_{\mathrm{e}}-k_{1} t
$$

Nonlinear forms of pseudo-second-order:

$$
Q_{t}=\frac{t}{1 / k_{2} Q_{\mathrm{e}}^{2}+t / Q_{\mathrm{e}}}
$$

Linear forms of pseudo-second-order:

$$
\frac{t}{Q_{t}}=\frac{1}{k_{2} Q_{\mathrm{e}}^{2}}+\frac{1}{Q_{\mathrm{e}}} t
$$

where $Q_{\mathrm{e}}$ and $Q_{t}$ are the adsorption capacity $\left(\mathrm{mg} \mathrm{g}^{-1}\right)$ at equilibrium time and at time $t(\mathrm{~min}), k_{1}$ and $k_{2}$ are pseudo-first order rate constant $\left(\mathrm{min}^{-1}\right)$, and pseudo-second order rate constant $(\mathrm{g}$ $\left.\mathrm{mg}^{-1} \min ^{-1}\right)$, respectively. 
Table 2 Adsorption kinetics values of Lcys-CNCs for $\mathrm{Hg}(\Perp)$

\begin{tabular}{|c|c|c|c|c|c|c|c|}
\hline Sample & \multicolumn{7}{|l|}{ Pseudo-first-order } \\
\hline Lcys-CNCs & 211 & 200 & 1.649 & 0.6340 & 21 & $2.61 \times 10^{-2}$ & 0.6641 \\
\hline \multicolumn{8}{|c|}{${ }^{a} Q_{\mathrm{e}}$,exp: experimental adsorption capacity. ${ }^{b} Q_{\mathrm{e}}$, cal: calculated adsorption capacity. } \\
\hline & \multicolumn{4}{|l|}{ Nonlinear form } & \multicolumn{3}{|l|}{ Linear form } \\
\hline Sample & $Q_{\mathrm{e}}, \exp \left(\mathrm{mg} \mathrm{g}^{-1}\right)$ & $Q_{\mathrm{e}}, \mathrm{cal}\left(\mathrm{mg} \mathrm{g}^{-1}\right)$ & $k_{2}\left(\mathrm{~g} \mathrm{mg}^{-1} \min ^{-1}\right)$ & $R^{2}$ & $Q_{\mathrm{e}}, \mathrm{cal}\left(\mathrm{mg} \mathrm{g}^{-1}\right)$ & $k_{2}\left(\mathrm{~g} \mathrm{mg}^{-1} \mathrm{~min}^{-1}\right)$ & $R^{2}$ \\
\hline Lcys-CNCs & 211 & 210 & $1.34 \times 10^{-2}$ & 0.8947 & 209 & $1.08 \times 10^{-2}$ & 0.9994 \\
\hline
\end{tabular}

Adsorption kinetics values of Lcys-CNCs for $\mathrm{Hg}$ (II) are summarized in Table 2. The equilibrium adsorption capacity calculated based on the pseudo-second order model is closer to the experimental data than the pseudo-first order model, and correlation coefficients $\left(R^{2}\right)$ value for the pseudo-second order model is much better than the pseudo-first order model. Therefore, the adsorption kinetics was accurately described by a pseudo-second order model, suggesting that the adsorption of $\mathrm{Hg}$ (II) on Lcys-CNCs was mainly through chemical reactive adsorption between active sites (thiol group and amino group) of Lcys-CNCs and $\mathrm{Hg}$ (II) ions. ${ }^{43}$

\subsection{Adsorption isotherm}

The initial concentration of mercury ion is also an important factor in affecting the mercury uptake capacity of Lcys-CNCs. The effect of initial ion concentration (71-502 $\left.\mathrm{mg} \mathrm{L}^{-1}\right)$ on the adsorption capacity of $\mathrm{Hg}$ (II) was shown in Fig. 4a. The adsorption values increased with the enhancing of initial concentration of mercury ion, then attained a platform. The equilibrium adsorption isotherm can help explain the relationship of metal ions and adsorbent molecule. The Langmuir and Freundlich models (Fig. 4b-d) are two well-known isothermal adsorption models, and their models are shown as the following equations.

Nonlinear forms of Langmuir:

$$
Q_{\mathrm{e}}=\frac{Q_{\mathrm{m}} b C_{\mathrm{e}}}{1+b C_{\mathrm{e}}}
$$

Linear forms of Langmuir:

$$
\frac{C_{\mathrm{e}}}{Q_{\mathrm{e}}}=\frac{1}{b Q_{\mathrm{m}}}+\frac{C_{\mathrm{e}}}{Q_{\mathrm{m}}}
$$

Nonlinear forms of Freundlich:

$$
Q_{\mathrm{e}}=K_{\mathrm{F}} C_{\mathrm{e}^{\frac{1}{n}}}^{\frac{1}{2}}
$$

Linear forms of Freundlich:

$$
\ln \left(Q_{\mathrm{e}}\right)=\ln \left(K_{\mathrm{F}}\right)+\frac{1}{n} \ln \left(C_{\mathrm{e}}\right)
$$

where $C_{\mathrm{e}}, Q_{\mathrm{e}}$ and $Q_{\mathrm{m}}$ are the equilibrium concentration $(\mathrm{mg}$ $\left.\mathrm{L}^{-1}\right)$, the equilibrium adsorption capacity of the adsorbent ( $\mathrm{mg}$ $\mathrm{g}^{-1}$ ) and the maximum adsorption capacity $\left(\mathrm{mg} \mathrm{g}^{-1}\right)$, respectively. $K_{\mathrm{F}}$ and $b$ are the Freundlich and Langmuir constant, and $n$ is the heterogeneity factor.

The calculated parameters for Langmuir and Freundlich isotherm models of Lcys-CNCs are summarized in Table 3. The $R^{2}$ of Langmuir model are higher than Freundlich model, and the maximum adsorption values calculated by Langmuir model are close to the experimental data $\left(923 \mathrm{mg} \mathrm{g}^{-1}\right)$, and which is higher than most of the adsorbents reported in the literatures (Table 4). Therefore, the equilibrium adsorption isotherm data was fitted with Langmuir model, suggesting that $\mathrm{Hg}$ (II) adsorption on adsorbent is a monolayer coverage. For the
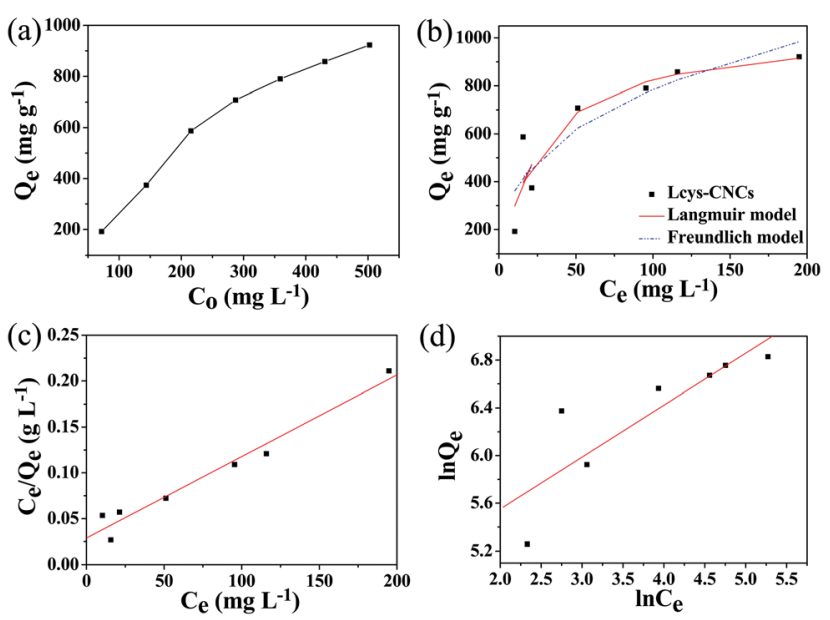

Fig. 4 Effect of initial ion concentration on the adsorption capacity of $\mathrm{Hg}($ (I) (a); nonlinear forms of Langmuir isotherm model and Freundlich isotherm model for adsorption of Hg(॥) by Lcys-CNCs (b); linear forms of Langmuir isotherm model (c) and Freundlich isotherm model (d) (3 h, pH 5, $25^{\circ} \mathrm{C}, 71-502 \mathrm{mg} \mathrm{L}^{-1}$ ). 
Table 3 Langmuir and Freundlich values of Lcys-CNCs for Hg(॥)

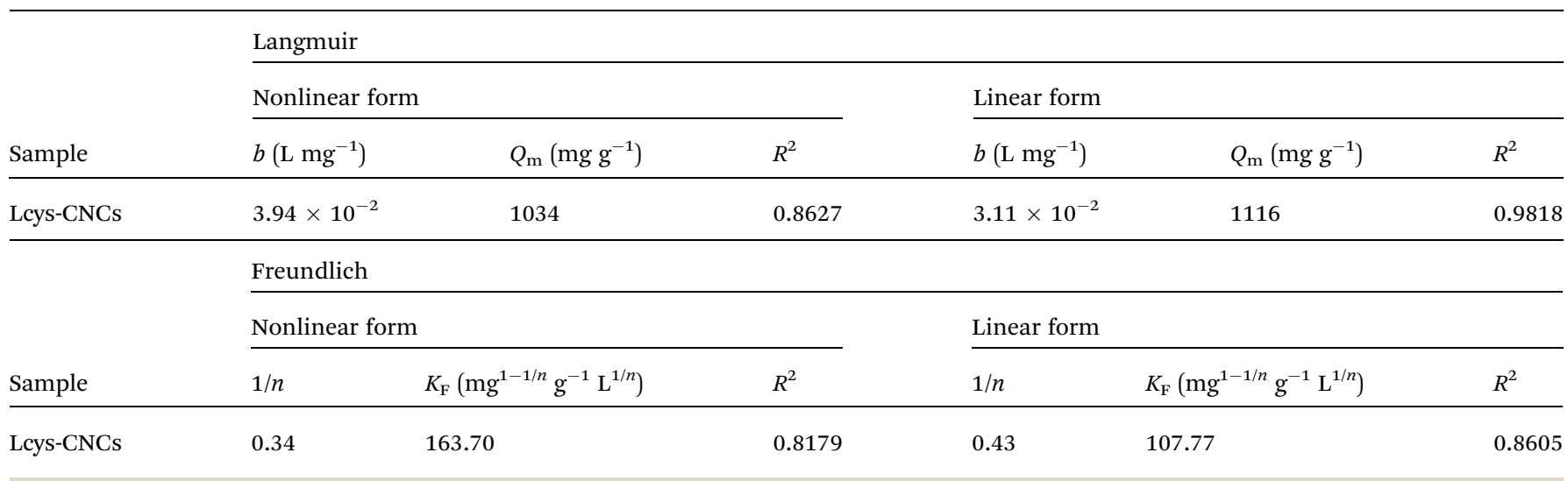

Langmuir model, the values of $b(0.03-0.04)$ lie between 0 and 1 , indicating a favorable adsorption process. ${ }^{44}$

\subsection{Adsorption mechanism}

The surface microstructure of Lcys-CNCs was analyzed by SEM (Fig. 5a) and it shown that rodlike shape with length of $228 \pm$ $57 \mathrm{~nm}$ and width of $25 \pm 6 \mathrm{~nm}$ (Fig. S2, Table S2 $\dagger$ ). Although the morphology of $\mathrm{Hg}$ (II)-loaded Lcsy-CNCs (Fig. 5b) was still rodlike shape, there was a accumulation phenomenon, and the width (35 $\pm 11 \mathrm{~nm}$ ) was obviously broader due to adsorptions of $\mathrm{Hg}$ (II) on the same Lcys-CNCs or between with Lcys-CNCs. ${ }^{46}$ It was further proved by EDX spectrum of $\mathrm{Hg}$ (II)-loaded Lcsy-CNCs (Fig. 5c) that Lcys-CNCs effectively adsorbed a large amount of mercury ions.

When Lcys-CNCs were added to the mercury ion solution, the proposed mechanism of $\mathrm{Hg}$ (II) uptake onto Lcys-CNCs was shown in Fig. 5d. Compared to the common adsorbents, nanoadsorbent Lcys-CNCs have higher specific surface area (72.10 $\mathrm{m}^{2} \mathrm{~g}^{-1}$ ). The high specific surface area of Lcys-CNCs is beneficial to contact with more mercury ions. Moreover, the negative charge of Lcys-CNCs could further accelerate the arrival of mercury ions on the surface of the adsorbent by electrostatic interaction. Then, the lone pairs of electrons of the $\mathrm{S}, \mathrm{N}$ and $\mathrm{O}$ elements on the Lcys-CNCs could rapidly complex mercury ions. The abundant active groups (amino and thiol groups) of LcysCNCs indicate that it has the excellent adsorption capacity. Therefore, Lcsy-CNCs could rapidly and efficiently adsorb

Table 4 Comparison of adsorption capacity with other reported adsorbents

\begin{tabular}{lll}
\hline Adsorbent & $Q_{\max }\left(\mathrm{mg} \mathrm{g}^{-1}\right)$ & Ref. \\
\hline TO-NFC-Si-SH & 718 & 38 \\
P(MB-IA)-g-MNCC & 240 & 45 \\
Guanyl-modified celluloseI & 48 & 14 \\
Mercaptobenzothiazole impregnated & 204 & 20 \\
cellulose & & \\
Lcys-CNCs & 923 & This work
\end{tabular}

mercury ions from aqueous solution, through complexation and electrostatic attraction.

\subsection{Selective studies}

In contaminated water, mercury ions often coexist with a large number of other metal ions. The adsorption capacity of mercury ion can also be affected by the presence of other coexisting metal ions. In order to investigate the selectivity of Lcsy-CNCs for mercury ions, $\mathrm{Pb}$ (II), $\mathrm{Cd}$ (II), $\mathrm{Zn}$ (II) and $\mathrm{Cu}$ (II) were used as coexisting ions to investigate their effects on the adsorption capacity of mercury ions, as shown in Fig. 6a. The adsorption efficiency of Lcsy-CNCs is 87.4, 2.1, 18.9, 6.1 and 2.7\% for $\mathrm{Hg}$ (II), $\mathrm{Cd}(\mathrm{II}), \mathrm{Pb}(\mathrm{II}), \mathrm{Cu}(\mathrm{II})$ and $\mathrm{Zn}(\mathrm{II})$, respectively. For the other four kinds of metal ion species, Lcsy-CNCs can selectly adsorb mercury ions. This is mainly based on the Hard-Soft Acid-Base (HSAB) theory, mercury ion was soft acids, and thiol group of Lcsy-CNCs was soft base, mercury ions could form steady complex with thiol groups. ${ }^{47}$ Therefore, Lcys-CNCs would

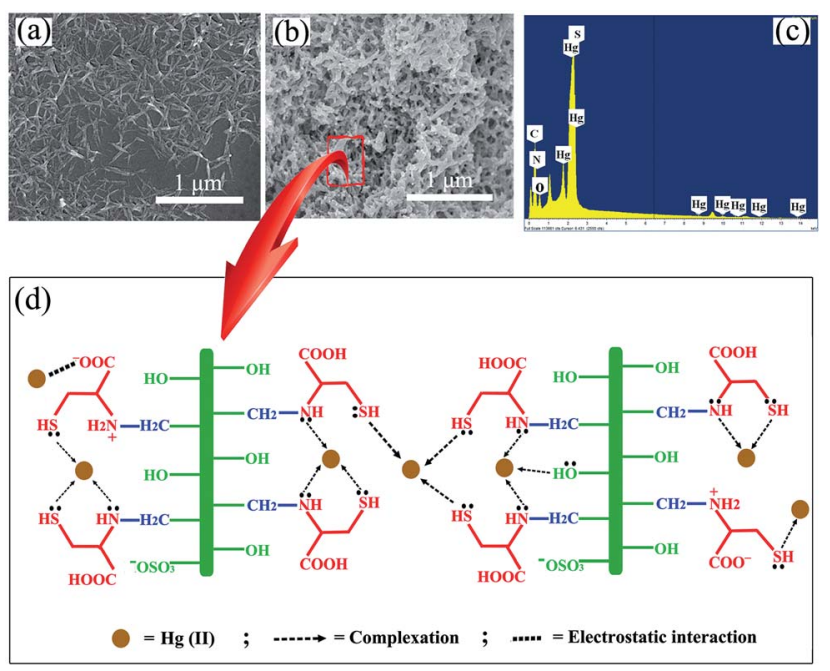

Fig. 5 SEM images of Lcsy-CNCs (a), and Hg(॥)-loaded Lcsy-CNCs (b). (c) EDX spectrum of $\mathrm{Hg}(\mathrm{II})$-loaded Lcsy-CNCs. (d) Illustration of the proposed interaction between Lcsy-CNCs and $\mathrm{Hg}\left({ }^{\prime \prime}\right)$. 

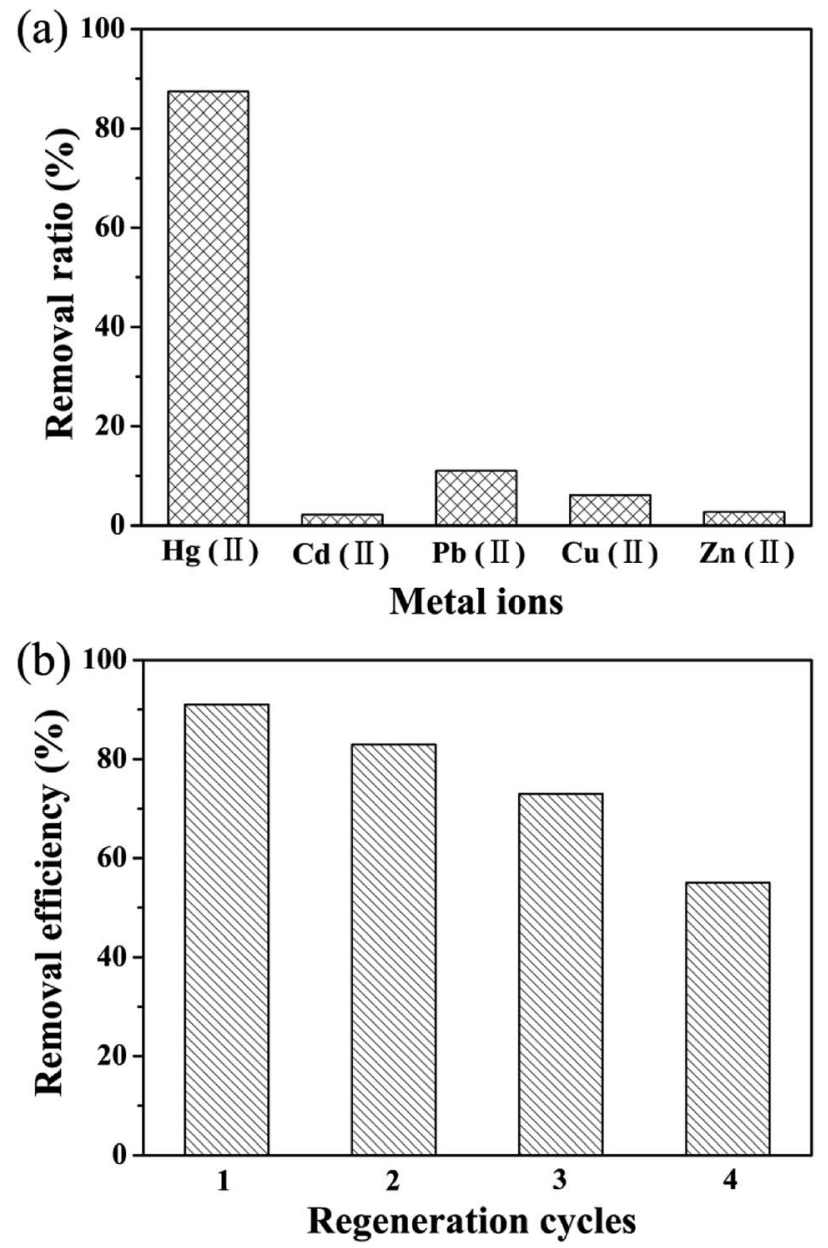

Fig. 6 (a) Selective adsorption results for mercury ions ( $3 \mathrm{~h}, \mathrm{pH} 5,25$ ${ }^{\circ} \mathrm{C}$ ); (b) reusability results for Lcsy-CNCs in $\mathrm{Hg}($ (I) solutions (3 h, $\mathrm{pH} 5,25$ $\left.{ }^{\circ} \mathrm{C}\right)$.

preferentially and more stably bind $\mathrm{Hg}$ (II) relative to other metal ions.

\subsection{Reusability test}

In practical applications, it is very necessary to investigate the regeneration performance of the adsorbent. The regeneration performance of Lcsy-CNCs was shown in Fig. 6b. After three cycles, the removal percentage of $\mathrm{Hg}$ (II) could still keep above $70 \%$, but after the fourth cycle, the removal percentage of $\mathrm{Hg}$ (II) for Lcsy-CNCs was reduced to $55 \%$.

\section{Conclusions}

A green biosorbent, L-cysteine modified cellulose nanocrystals (Lcys-CNCs), was successfully synthesized and applied to adsorb mercury ions from aqueous solutions. Lcys-CNCs show the excellent adsorption capacity for mercury ions. In the mercury ion solution with a concentration of $71 \mathrm{mg} \mathrm{L}^{-1}$, the adsorption of $\mathrm{Hg}(\mathrm{II})$ achieved adsorption equilibrium within $5 \mathrm{~min}$. The optimum $\mathrm{pH}$ value for $\mathrm{Hg}$ (II) removal was 5 . The adsorption of $\mathrm{Hg}$ (II) fitted Langmuir isotherm and pseudo- second order. The maximum adsorption value of Lcys-CNCs was up to $923 \mathrm{mg} \mathrm{g}^{-1}$. And Lcys-CNCs can selectively adsorb mercury ions in the presence of other coexisting metal ions. After three adsorption/desorption cycles, the removal percentage of $\mathrm{Hg}$ (II) could still keep above $70 \%$. Therefore, LcsyCNCs have great potential as a green biosorbent for highly efficient adsorption of mercury ions from aqueous solutions.

\section{Conflicts of interest}

There are no conflicts to declare.

\section{Acknowledgements}

This work was supported by the National Natural Science Foundation of China (Grant No. 21376041), (Grant No. 21076033), (Grant No. 21536002), (Grant No. 21878036).

\section{References}

1 N. Lubick and D. Malakoff, Science, 2013, 341, 1443-1445.

2 V. K. Gupta, I. Ali, T. A. Saleh, A. Nayak and S. Agarwal, $R S C$ Adv., 2012, 2, 6380-6388.

3 P. M. Bolger and B. A. Schwetz, N. Engl. J. Med., 2002, 347, 1735-1736.

4 T. A. Saleh, Desalin. Water Treat., 2016, 57, 10730-10744.

5 H. A. Sani, M. B. Ahmad and T. A. Saleh, RSC Adv., 2016, 6, 108819-108827.

6 C. P. Huang and D. W. Blankenship, Water Res., 1984, 18, 3746.

7 G. Blanchard, M. Maunaye and G. Martin, Water Res., 1984, 18, 1501-1507.

8 N. L. D. Filho, W. L. Polito and Y. Gushikem, Talanta, 1995, 42, 1031-1036.

9 F. Abadast, A. Mouradzadegun and M. R. Ganjali, New J. Chem., 2017, 41, 5458-5466.

10 U. Farooq, J. A. Kozinski, M. A. Khan and M. Athar, Bioresour. Technol., 2010, 101, 5043-5053.

11 N. Salamun, S. Triwahyono, A. A. Jalil, T. Matsuura and N. F. M. Salleh, RSC Adv., 2015, 5, 14129-14137.

12 C. Hou, D. Zhao, S. Zhang and Y. Wang, Colloid Polym. Sci., 2018, 296, 547-555.

13 S. Kumari and G. S. Chauhan, ACS Appl. Mater. Interfaces, 2014, 6, 5908-5917.

14 I. M. M. Kenawy, M. A. H. Hafez, M. A. Ismail and M. A. Hashem, Int. J. Biol. Macromol., 2018, 107, 1538-1549.

15 Y. Zhou, X. Hu, M. Zhang, X. Zhuo and J. Niu, Ind. Eng. Chem. Res., 2013, 52, 876-884.

16 J. D. Merrifield, W. G. Davids, J. D. Macrae and A. Amirbahman, Water Res., 2004, 38, 3132-3138.

17 J. Song, H. Oh, H. Kong and J. Jang, J. Hazard. Mater., 2011, 187, 311-317.

18 M. J. Melgar, J. Alonso and M. A. Garcia, Sci. Total Environ., 2007, 385, 12-19.

19 T. G. M. V. De Ven and A. Sheikhi, Nanoscale, 2016, 8, 1510115114. 
20 A. S. K. Kumar, S. Kalidhasan, V. Rajesh and N. Rajesh, Ind. Eng. Chem. Res., 2013, 52, 11838-11849.

21 B. Geng, H. Wang, S. Wu, J. Ru, C. Tong, Y. Chen, H. Liu, S. Wu and X. Liu, ACS Sustainable Chem. Eng., 2017, 5, 11715-11726.

22 Y. Wang, Y. Zhang, C. Hou, X. He and M. Liu, J. Taiwan Inst. Chem. Eng., 2016, 58, 283-289.

23 A. S. K. Kumar and S. Jiang, $R S C A d v .$, 2015, 5, 6294-6304.

24 N. Ballav, R. Das, S. Giri, A. M. Muliwa, K. Pillay and A. Maity, Chem. Eng. J., 2018, 345, 621-630.

25 L. Jin, W. Li, Q. Xu and Q. Sun, Cellulose, 2015, 22, 24432456.

26 S. M. A. S. Keshk, A. M. Ramadan and S. Bondock, Carbohydr. Polym., 2015, 127, 246-251.

27 U. Kim, M. Wada and S. Kuga, Carbohydr. Polym., 2004, 56, 7-10.

28 F. Azzam, M. Galliot, J. L. Putaux, L. Heux and B. Jean, Cellulose, 2015, 22, 3701-3714.

29 S. Ravi, P. Puthiaraj, K. H. Row, D. Park and W. Ahn, Ind. Eng. Chem. Res., 2017, 56, 10174-10182.

30 N. M. Bandaru, N. Reta, H. Dalal, A. V. Ellis, J. G. Shapter and N. H. Voelcker, J. Hazard. Mater., 2013, 261, 534-541.

31 A. Gupta, S. R. Vidyarthi and N. Sankararamakrishnan, J. Hazard. Mater., 2014, 274, 132-144.

32 S. Y. Oh, D. I. Yoo, Y. Shin and G. Seo, Carbohydr. Res., 2005, 340, 417-428.

33 A. R. Lokanathan, A. Nykanen, J. Seitsonen, L. Johansson, J. M. Campbell, O. J. Rojas, O. Ikkala and J. Laine, Biomacromolecules, 2013, 14, 2807-2813.
34 L. Tang, T. Li, S. Zhuang, Q. Lu, P. Li and B. Huang, ACS Sustainable Chem. Eng., 2016, 4, 4842-4849.

35 H. Kono, S. Yunoki, T. Shikano, M. Fujiwara, T. Erata and M. Takai, J. Am. Chem. Soc., 2002, 124, 7506-7511.

36 M. M. Ibrahim, W. K. Elzawawy and M. A. Nassar, Carbohydr. Polym., 2010, 79, 694-699.

37 H. Yang and T. G. M. V. De Ven, Cellulose, 2016, 23, 17911801.

38 B. Ram and G. S. Chauhan, Chem. Eng. J., 2018, 331, 587-596.

39 W. R. Knocke and L. H. Hemphill, Water Res., 1981, 15, 275282.

40 T. Dashman and G. Stotzky, Soil Biol. Biochem., 1982, 14, 447-456.

41 M. Arshadi, A. R. Faraji and M. J. Amiri, Chem. Eng. J., 2015, 266, 345-355.

42 R. Soury, M. Jabli, T. A. Saleh, W. S. AbdulHassan, E. SaintAman, F. Loiseau, C. Philouze and H. Nasri, RSC Adv., 2018, 8, 20143-20156.

43 E. Repo, J. K. Warchol, T. A. Kurniawan and M. Sillanpaa, Chem. Eng. J., 2010, 161, 73-82.

44 V. Vadivelan and K. V. Kumar, J. Colloid Interface Sci., 2005, 286, 90-100.

45 T. S. Anirudhan and F. Shainy, J. Colloid Interface Sci., 2015, 456, 22-31.

46 H. Dong, J. F. Snyder, K. S. Williams and J. W. Andzelm, Biomacromolecules, 2013, 14, 3338-3345.

47 R. G. Pearson, J. Chem. Educ., 1968, 45, 581-587. 